\title{
Rayleigh-Ritz variational method with suitable asymptotic behaviour
}

Research Article

\author{
Francisco M Fernández* and Javier Garcia
}

INIFTA (UNLP, CCT La Plata-CONICET), División Química Teórica Diag. 113 y 64 (S/N), Sucursal 4, Casilla de Correo 161900 La Plata, Argentina

Received 14 December 2013; accepted 24 April 2014

\begin{abstract}
:
This paper considers the Rayleigh-Ritz variational calculations with non-orthogonal basis sets that exhibit the correct asymptotic behaviour. This approach is illustrated by constructing suitable basis sets for onedimensional models such as the two double-well oscillators recently considered by other authors. The rate of convergence of the variational method proves to be considerably greater than the one exhibited by the recently developed orthogonal polynomial projection quantization.
\end{abstract}

PACS (2008): 03.65.Ge

Keywords: $\quad$ Rayleigh-Ritz method $\cdot$ asymptotic behaviour $\cdot$ anharmonic oscillators $\cdot$ rate of convergence $\bullet$ orthogonal polynomial projection quantization

(c) Versita Sp. z o.o.

\section{Introduction}

In a recent paper Handy and Vrinceanu [1] proposed a method for the calculation of energy eigenvalues that is based on the projection of the bound-state wavefunction onto sets of orthogonal polynomials. The approach named orthogonal polynomial projection quantization (OPPQ) proved to be rapidly converging and more stable than the Hill determinant methods.

Among other models, the authors considered the sextic $V_{S}(x)=x^{6}-4 x^{2}$ and quartic $V_{Q}(x)=x^{4}-5 x^{2}$ two-well oscillators. The bound states behave asymptotically as $\psi(x) \sim e^{-x^{4} / 4}$ and $\psi(x) \sim e^{-|x|^{3} / 3}$ in the former and latter case, respectively. Handy and Vrinceanu [1] chose the

*E-mail: fernande@quimica.unlp.edu.ar reference functions $R_{G}(x)=e^{-x^{2} / 2}$ and $R_{T T}(x)=e^{-x^{4} / 4}$ and showed that the latter is preferable for $V_{S}(x)$ while the former is more convenient for $V_{Q}(x)$. In fact, $R_{T T}(x)$ exhibits the correct asymptotic behaviour for the potential $V_{S}(x)$. At first sight, it appears to be surprising that the authors did not try the reference function $R(x)=e^{-|x|^{3} / 3}$, which is expected to be suitable for the quartic double well, since their approach permits the use of arbitrary nonanalytic positive reference functions [1].

The purpose of this paper is to show that it is quite straightforward to apply the variational Rayleigh-Ritz method (RRM) [2] with a basis set that exhibits the correct asymptotic behaviour of the eigenfunctions for the models discussed above. In addition to this, it is worthwhile to compare the well known, extremely reliable and widely used RRM with the recently developed OPPQ.

In section 2 the basis sets are developed with suitable asymptotic behaviours for some general one-dimensional 
models. In section 3 the eigenvalues are calculated for the oscillators $V_{S}(x)$ and $V_{Q}(x)$ with three basis sets having different asymptotic behaviours, including the correct one for each model. The RRM and OPPQ results are compared for two models and two basis sets. Finally, section 4 summarizes the main conclusions of the paper.

\section{Basis functions with suitable asymptotic behaviour}

This section shows how a non-orthogonal basis set is built with the appropriate asymptotic behaviour at infinity. This generalizes a procedure proposed recently by Fernández [3] for a particular case. For simplicity, this paper focuses on the one-dimensional eigenvalue equation

$$
-\psi^{\prime \prime}(x)+V(x) \psi(x)=E \psi(x)
$$

and assumes that

$$
\lim _{|x| \rightarrow \infty} x^{-2 k} V(x)=a>0
$$

Under such conditions, the eigenfunctions behaves asymptotically as

$$
\begin{aligned}
\psi(x) & \sim e^{-\left|S_{k}(x)\right|} \\
S_{k}(x) & =\frac{\sqrt{a}}{k+1} x^{k+1} .
\end{aligned}
$$

The following cases are considered:

Case 1: Parity-invariant potential $V(-x)=V(x)$.

a) $k$ even. The non-orthogonal basis set is of the form

$$
\begin{aligned}
& f_{j}(x)=|x|^{j} e^{-\left|S_{k}(x)\right|}, j=0,2,3, \ldots \text { even states, } \\
& f_{j}(x)=x|x|^{j} e^{-\left|S_{k}(x)\right|}, j=0,1, \ldots \text { odd states, }
\end{aligned}
$$

b) $k$ odd. In this case,

$$
\begin{aligned}
f_{j}(x) & =x^{2 j+s} e^{-S_{k}(x)}, j=0,1, \ldots \\
s & =\left\{\begin{array}{c}
0 \text { even states } \\
1 \text { odd states }
\end{array}\right.
\end{aligned}
$$

Case 2: Asymmetric potential $V(-x) \neq V(x)$. The basis set is

$$
f_{j}(x)=x^{j} e^{-\left|S_{k}(x)\right|}, j=0,1, \ldots
$$

For completeness, two examples by Handy and Vrinceanu [1] are considered in this paper:

$$
V_{Q}(x)=x^{4}-5 x^{2}
$$

and

$$
V_{S}(x)=x^{6}-4 x^{2}
$$

with asymptotic behaviours given by $S_{2}(x)=x^{3} / 3$ and $S_{3}(x)=x^{4} / 4$, respectively. However, Handy and Vrinceanu chose reference functions associated to $S_{3}(x)$ and $S_{1}(x)=x^{2} / 2$ for the two models.

From RRM [2], the eigenvalues are obtained approximately from the roots of the secular determinant

$$
|\mathbf{H}-E \mathbf{S}|=0,
$$

where $\mathrm{H}$ and $\mathrm{S}$ are $\mathrm{N} \times N$ square matrices with elements

$$
H_{i j}=\left\langle f_{i}|\hat{H}| f_{j}\right\rangle, S_{i j}=\left\langle f_{i} \mid f_{j}\right\rangle,
$$

and $\hat{H}=\hat{p}^{2}+V(x)$. Once the approximate eigenvalues have been obtained, the eigenvectors can be calculated from the secular equation

$$
(\mathrm{H}-E S) \mathrm{C}=\mathbf{0}
$$

where $C$ is an $N \times 1$ column matrix with the coefficients $c_{j}$ of the variational trial function.

The application of this approach to the Schrödinger equation with a parity-invariant potential is particularly simple because it can restrict the calculation of the matrix elements to the half line $x>0$ [3]. Since all the matrix elements reduce to integrals of the form

$$
\langle f \mid g\rangle=\int_{0}^{\infty} f(x) g(x) d x
$$

then the absolute value of the coordinate does not have to be explicitly taken into account when $k$ is even.

\section{Results}

Firstly, the effect of the asymptotic behaviour of the basis set (4) and (5) on the rate of convergence of the RRM is verified. A reasonable estimate of the rate of convergence is the logarithmic error $L_{N}=\log \left|E_{n}^{(a p p)}-E_{n}^{(R P M)}\right|$ where $E_{n}^{(a p p)}$ is the eigenvalue calculated by any of the methods described in this paper and $E_{n}^{(R P M)}$ is a very accurate result obtained by means of the Riccati-Padé method $[4,5]$. Figure 1 shows $L_{N}$ for the first four eigenvalues of (7) calculated by means of the RRM with the functions $S_{1}(x), S_{2}(x)$ and $S_{3}(x)$ in terms of the number of basis functions $N$. The rate of convergence decreases according to $S_{2}(x)>S_{1}(x)>S_{3}(x)$; that is to say, the 

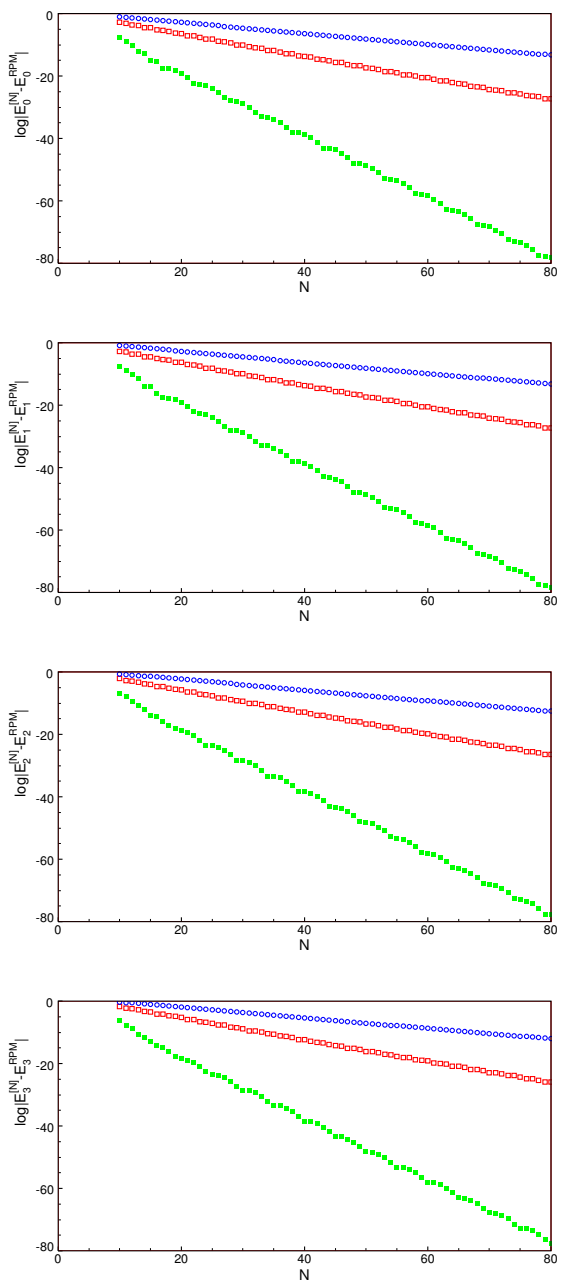

Figure 1. Logarithmic errors for the first four eigenvalues of the double well $V_{Q}(x)$ calculated by means of the basis sets with $S_{1}(x)$ (squares, red) $S_{2}(x)$ (filled squares, green) and $S_{3}(x)$ (circles, blue)

RRM converges more rapidly when choosing the correct asymptotic behaviour $S_{2}(x)$. In all the cases, the rate of convergence is exponential as follows from the fact that $L_{N}=0.518-0.991 \mathrm{~N}, L_{N}=0.427-0.352 \mathrm{~N}$ and $L_{N}=0.689-0.177 \mathrm{~N}$, respectively.

Figure 2 shows that the rate of convergence of the RRM for the potential (8) is also exponential but in this case it decreases according to $S_{3}(x)>S_{2}(x)>S_{1}(x)$. Once again the greater rate of convergence is given by the correct asymptotic behaviour $S_{3}(x)$. Besides, the second inequality appears to be reasonable because $S_{2}(x)$ is closer to the correct asymptotic behaviour than $S_{1}(x)$.

At this point it is worthwhile to compare the rate of convergence of the simple, well known and reliable RRM [2] and
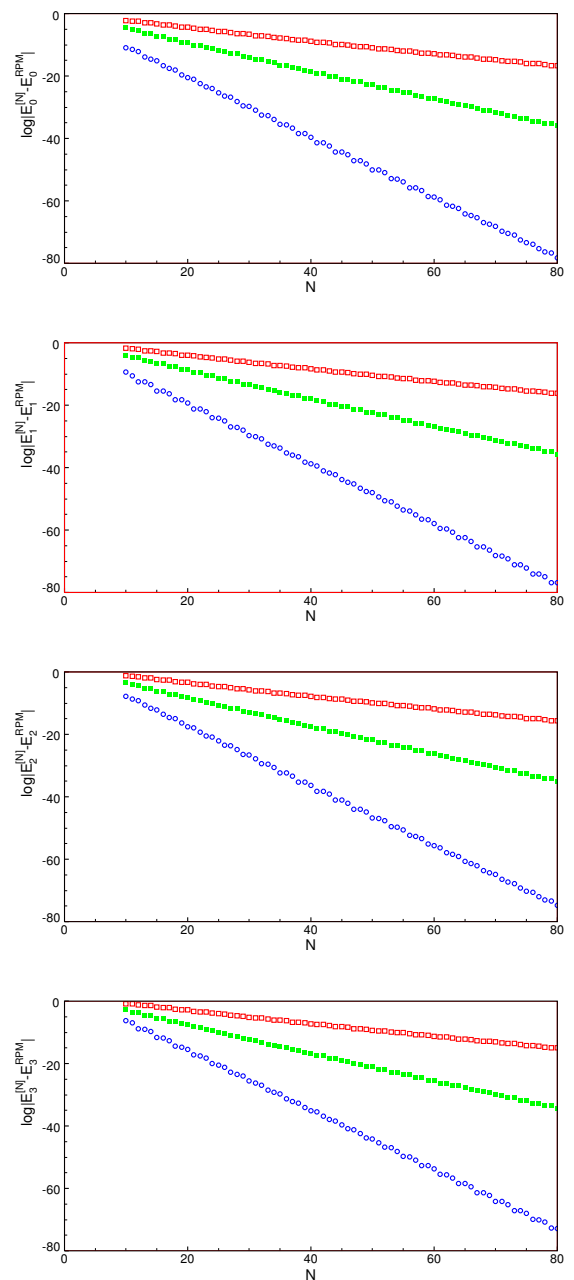

Figure 2. Logarithmic errors for the first four eigenvalues of the double well $V_{S}(x)$ calculated by means of the basis sets with $S_{1}(x)$ (squares, red) $S_{2}(x)$ (filled squares, green) and $S_{3}(x)$ (circles, blue)

the rather more elaborate OPPQ using the same basis set in both approaches. Handy and Vrinceanu [1] choose the reference function $R_{G}(x)=e^{-x^{2} / 2}$ for the PT-symmetric potential

$$
V(x)=i x^{3}
$$

and here the function $S_{1}(x)$ is chosen for the RRM. More precisely, instead of the non-orthogonal basis set (5), this method uses the eigenfunctions of the harmonic oscillator that are truly consistent with the orthogonal Hermite polynomials used by those authors. Figure 3 shows $L_{N}$ for the first four eigenvalues calculated by both methods. It clearly shows that the RRM rate of convergence is noticeably greater that the OPPQ one. 
The better performance of the RRM is not restricted to the PT-symmetric cubic oscillator; this approach converges more rapidly for the two other models discussed above. For example, Figure 4 compares the logarithmic errors of both approaches for the eigenvalues of the double-well oscillator (8). In this case, the method chooses the basis set with the correct asymptotic behaviour $S_{3}(x)$ for the RRM and the OPPQ results for $R_{T T}(x)=e^{-x^{4} / 4}$ [1]. The differ- ence between the convergence rates of both approaches is even more dramatic for this oscillator. However, it makes more sense for small $N$ because the number of significant digits of the OPPQ results reported by the authors is rather too small for a fair comparison at large $N$.

Finally, it is worthwhile to show the RPM eigenvalues chosen as a reference for the calculation of the logarithmic errors. Specifically,

$$
\begin{aligned}
& E_{0}=-3.410142761239829475297709653521909198712339047564881868937911775329611301715294 \\
& E_{1}=-3.2506753622892359802285137755477368771546011476394241429953014335680690809034749688022953825298 \\
& E_{2}=0.638919563783838124491010103332504264852401329058137207433367771840730088316019330941500824 \\
& E_{3}=2.5812162706174514809779380656962090234197947974759598949291704975284539346710703866627200928172
\end{aligned}
$$

for (7)

$$
\begin{aligned}
& E_{0}=-0.523268622127552239416169497190784061165634222518711069953854385633821213450649003542309 \\
& E_{1}=1.00576834022554481670604083074777604686886504417542730471341100873617568288708176003637 \\
& E_{2}=5.374970008840044994060514769418235325821754311501338177585996687355671683247232390293 \\
& E_{3}=10.572585044585912113906061555314011464842213880057529217715660995992776130576146017312(15)
\end{aligned}
$$

for (8) and

$$
\begin{aligned}
& E_{0}=1.156267071988113293799219177999951 \\
& E_{1}=4.1092287528096515358436684785613 \\
& E_{2}=7.5622738549788280413518091106314827208 \\
& E_{3}=11.314421820195804402233783948426989
\end{aligned}
$$

for (13). These quite accurate results may be used as benchmarks for other approaches.

\section{Conclusions}

This paper shows that it is not difficult to introduce the correct asymptotic behaviour of the wavefunction into the RRM variational trial function, specially if the potential is parity invariant. Present results clearly show that the correct asymptotic behaviour increases the rate of convergence of the approach dramatically. In principle, the same strategy can be implemented through the appropriate OPPQ reference function but it has not yet been tried for the case of $k$ even [1].
The rate of convergence for RRM is shown to be considerably greater than that for the OPPQ (with the reservations already indicated above). In addition, the former approach is simpler and more straightforward. The integrals that appear in both approaches are basically the same and can in principle be calculated by the same algorithms. In this paper, only the results for two oscillators and two basis sets with different asymptotic behaviours are compared but the trend is exactly the same for the other possible combinations of model and basis set.

The reason why the RRM rate of convergence is so much greater than for OPPQ is presently unknown to the authors. It would be of great benefit for the OPPQ results to be expressed with comparable accuracy. It is already known that, in the case of the Hermitian Hamiltonians, 

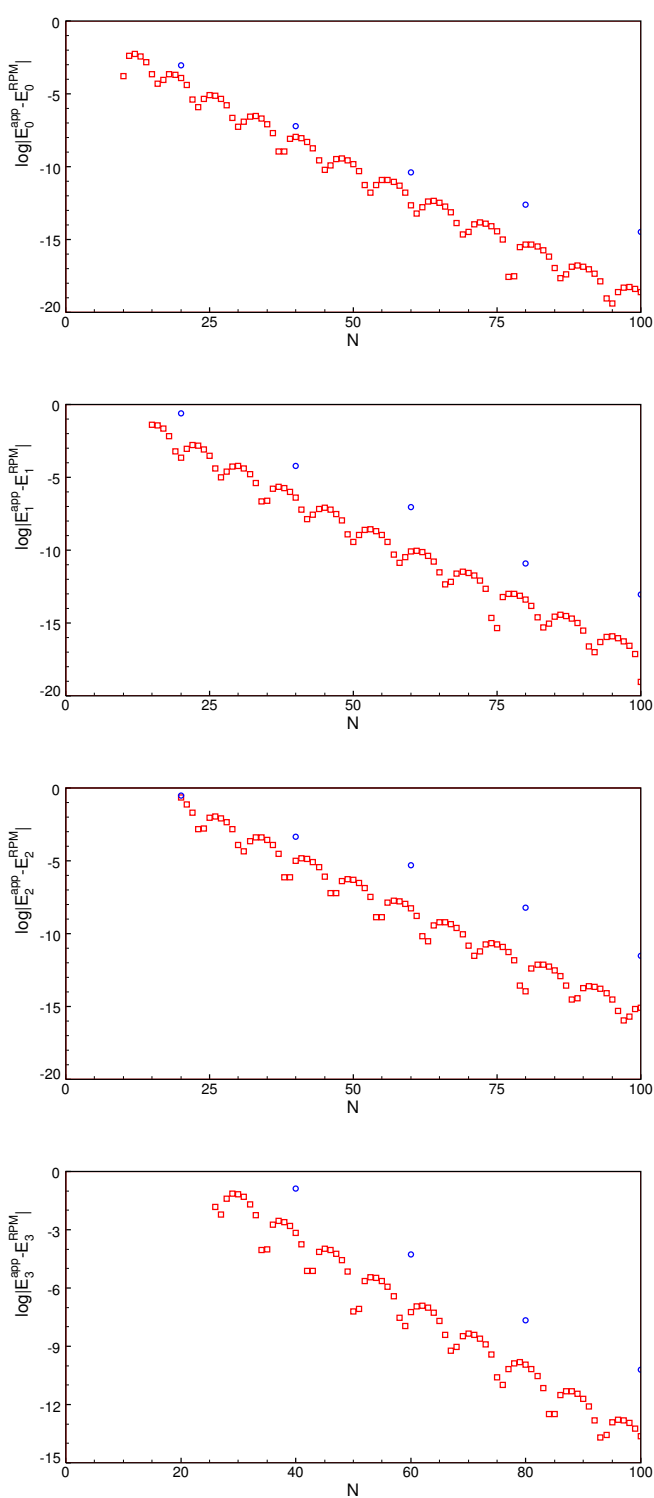

Figure 3. Logarithmic errors for the first four eigenvalues of the PTsymmetric potential (13) calculated by means of the RRM (squares, red) and the OPPQ method (circles, blue) the former approach exhibits the additional advantage that its approximate eigenvalues tend to the exact ones from above. On the other hand, the OPPQ eigenvalues do not appear to exhibit any bounding property.

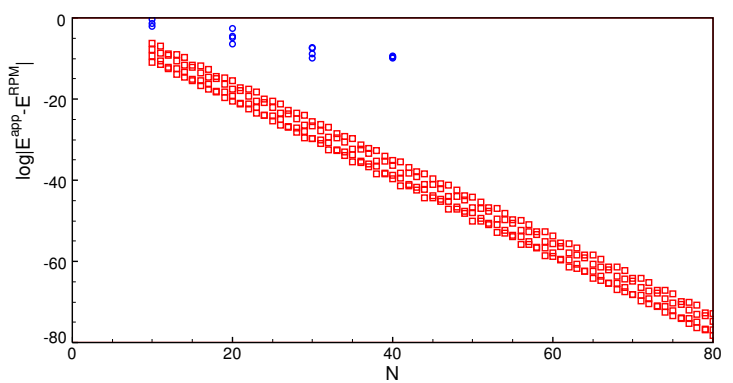

Figure 4. Logarithmic errors for the first four eigenvalues of the double well $V_{S}(x)$ calculated by means of the RRM (squares, red) and the OPPQ method (circles, blue)

\section{Acknowledgments}

This report has been financially supported by PIP No. 11420110100062 (Consejo Nacional de Investigaciones Cientificas y Tecnicas, República Argentina)

\section{References}

[1] C. N. Handy, D. Vrinceanu, J. Phys. A 46, 135202 (2013)

[2] F. L. Pilar, Elementary Quantum Chemistry (McGrawHill, New York, 1968)

[3] F. M. Fernández, Cent. Eur. J. Phys. 11, 470 (2013). arXiv:1204.0229 [math-ph]

[4] F. M. Fernández, Q. Ma, R. H. Tipping, Phys. Rev. A 39, 1605 (1989)

[5] F. M. Fernández, Q. Ma, R. H. Tipping, Phys. Rev. A 40, 6149 (1989) 